\title{
Dynamic Stress Factor (DySF): A Significant Predictor of Severe Hypoglycemic Events in Children with Type 1 Diabetes
}

\author{
Rawlings RA ${ }^{1,2}$, Yuan $\mathrm{L}^{3,4}$, Shi $\mathrm{H}^{5}$, Brehm $\mathbf{W}^{6}$, Pop-Busui $\mathbf{R}^{6,7}$ and Nelson $\mathrm{PW}^{1 *}$
}

${ }^{1}$ Center for Computational Medicine and Bioinformatics, University of Michigan, USA

${ }^{2}$ Departments of Biophysics, University of Michigan, USA

${ }^{3}$ Departments of Mathematics, University of Michigan, USA

${ }^{4}$ University of Michigan Program in Informatics, University of Michigan, USA

${ }^{5}$ University of Michigan Medical School, USA

${ }^{6}$ Brehm Center for Diabetes Research, University of Michigan, USA

${ }^{7}$ Internal Medicine, Division of Metabolism, Endocrinology and Diabetes, University of Michigan, USA

\begin{abstract}
Hemoglobin $\mathrm{A} 1 \mathrm{c}(\mathrm{HbA} 1 \mathrm{c})$ is the current standard used in the clinical treatment of patients with diabetes. However, it has been shown that patients with similar $\mathrm{HbA} 1 \mathrm{c}$ values may have widely different fluctuations in blood glucose values over the same period of time, including time spent in hyper- and/or hypo-glycemia. Hence, there exists a need for quantitative measures that can supplement $\mathrm{HbA} 1 \mathrm{c}$ in managing patients with diabetes. We introduce and compare the Dynamic Stress Factor, DySF, a newly developed metric that quantifies glycemic volatility based on patient-specific glucose transition density profiles with $\mathrm{HbA} 1 \mathrm{c}$ and with currently used glucose variability metrics in predicting severe hypoglycemia in children with type 1 diabetes. DySF, the daily weighted number of large monotonic glycemic transitions that occur within one hour, was calculated for 441 total subjects with type 1 diabetes (146 children, aged 8-14 yrs) to assess the magnitude and frequency of glucose transitions per day. Severe hypoglycemic episodes (HE) were quantified for all subjects and evaluated against $\mathrm{HbA} 1 \mathrm{c}$ and existing measures of glucose variability, including SD, MAGE, MODD, and CONGA using logistic regression models. DySF was found to be a predictor of severe HE in children $(p=0.018)$ with the likelihood of a child, aged 8-14 yrs, experiencing severe hypoglycemia increasing by up to $20 \%$ with decreasing values of up to $60 \%$ of DySF. Patients of any age who had one or multiple severe hypoglycemic episodes had on average a lower DySF when compared to those with no HE. Additionally, when considering mean glucose levels, DySF/mean was a preliminary predictor of severe $\mathrm{HE}$ in patients with $\mathrm{HbA1c} \leq 6.5 \%(p=0.062)$. DySF is a dynamic, quantitative, measure of daily glucose "volatility" that separates patients, within the same strata of HbA1c, into visually distinct patient profiles. DySF can be used as a preliminary predictor of clinically severe hypoglycemia in children and "well-controlled" patients with $\mathrm{HbA} 1 \mathrm{c} \leq 6.5 \%$.
\end{abstract}

Abbreviations: CGM: Continuous Glucose Monitor; DySF: Dynamic Stress Factor; MAGE: Mean Amplitude of Glucose Excursions; MODD: Mean of Daily Differences; CONGA: Continuous Overall Net Glycemic Action, SMBG: Self-Monitored Blood Glucose

\section{Introduction}

Emerging evidence suggests that chronic wide glucose fluctuations increase the risk of severe hypoglycemia and may be instrumental for the development of chronic diabetes complications. Therefore, development of sensitive tools to analyze blood glucose (BG) fluctuations and guide appropriate therapeutic changes to blunt wide BG excursions will have a critical role in preventing acute and chronic complications and improve quality of life in diabetic patients.

Continuous glucose monitors (CGMs) that record glucose levels at short, regular intervals throughout the day afford patients and physicians the flexibility to track glucose trends, evaluate frequency and severity of hypoglycemia including during nocturnal patterns, and assess individualized response to exercise and various other stressors. As the use of CGMs increases, conclusions previously drawn from single monitor blood glucose (SMBG) data can be tested against this more robust data set to guide optimization in individualized insulin regimens, to effectively prevent severe hypoglycemia, to blunt hyperglycemic peaks in response to meals and other stressors, and to evaluate the longer term consequences of glucose variability on the development of diabetic complications.

Traditionally, HbAlc is used to assess glycemic control and risk of complications in patients with diabetes [1,2]. However, emerging evidence suggest that glucose variability may also play an important role in assessing the risk for hypoglycemia and/or in the development of microvascular complications and cardiovascular disease [3-5] via several mechanisms including its role in oxidative stress and vascular pathology [6-8]

Several metrics to quantify glucose variability have been employed to date [9-12]. However, until recently the only data available for such studies were obtained through five- or seven-point single monitor profiles that provided only a restricted view of a patient's glucose dynamics over 24 hours $[1,13]$. These discrete glucose measurements are limiting in both the amount of information available for the analysis of glycemic variability and the methods by which variability can be examined.Metrics currently used to quantify glucose variability include, but are not limited to, standard deviation (SD), mean amplitude of glycemic excursions (MAGE) [14], mean of daily differences (MODD) [15], and continuous overall net glycemic action (CONGA(n)) [16],

*Corresponding author: Patrick Nelson, Center for Computational Medicine and Bioinformatics, 100 Washtenaw Ave, University of Michigan, Ann Arbor, Ml 48109-1055 USA, Tel: (734) 763-3408; Fax: (734) 615-6553; E-mail: pwn@umich.edu

Received January 17, 2012; Accepted February 24, 2012; Published February 28, 2012

Citation: Rawlings RA, Yuan L, Shi H, Brehm W, Pop-Busui R et al. (2012) Dynamic Stress Factor (DySF): A Significant Predictor of Severe Hypoglycemic Events in Children with Type 1 Diabetes. J Diabetes Metab 3:177 doi:10.4172/2155 6156.1000177

Copyright: (c) 2012 Rawlings RA, et al. This is an open-access article distributed under the terms of the Creative Commons Attribution License, which permits unrestricted use, distribution, and reproduction in any medium, provided the original author and source are credited. 
Citation: Rawlings RA, Yuan L, Shi H, Brehm W, Pop-Busui R et al. (2012) Dynamic Stress Factor (DySF): A Significant Predictor of Severe Hypoglycemic Events in Children with Type 1 Diabetes. J Diabetes Metab 3:177. doi:10.4172/2155-6156.1000177

and more recently standard deviation rate of change (SDRC), average absolute rate of change (AARC) [17], glucose error grid analysis (CGEGA), and prediction-error grid analysis (PRED-EGA) [18]. None of these, however, fully address the issue of glucose volatility in the context of hypoglycemia.

We have previously reported the development of the CGMGUIDE [19], an easy-to-use tool, that provides researchers and clinicians with a superior assessment of a patient's glucose landscape. The interface calculates and displays multiple metrics from inputted CGM data, offering not only a multifaceted approach to studying glucose variability, but also a means to investigate variability with more information-rich data sets.

Here, we report a new sensitive metric, DySF, which was developed with the CGM-GUIDE, which performs superiorly in quantifying glucose volatility by taking into account the speed and magnitude of glycemic excursions between clinically-defined states. DySF employs the recently developed transition density profile from CGM-GUIDE ${ }^{\complement}$ [19], which analyzes glucose excursions and transitions across different glycemic ranges, to predict the likelihood of onset of severe hypoglycemic episodes in a cohort of patients with type 1 diabetes. Based on continuous glucose dynamics, DySF is thereby a measure of a patient's daily glucose "volatility".

\section{Research Design and Methods}

We analyzed publicly archived CGM data from the Juvenile Diabetes Research Foundation (JDRF) Continuous Glucose Monitoring Randomized Trial [20]. Trial protocol has been described previously in detail [20]. Briefly, the enrollment criteria were children and adults with type 1 diabetes mellitus (T1DM) for more than 1 year (aged 8 to $85 \mathrm{yrs}$ ), use of either an insulin pump or at least three daily insulin injections, and $\mathrm{HbA} 1 \mathrm{c}<10.0 \%$.

This analysis used CGM data and HbA1c levels collected at baseline from 441 T1DM patients with complete demographic and CGM data. Of the 441 patients analyzed, $32.4 \%$ were aged $8-14$ yrs, $30.8 \%$ were aged $15-24$ yrs and, $36.7 \%$ were $\geq 25$ yrs. Patients were stratified into approximately equal percentages of subjects with $\mathrm{HbA} 1 \mathrm{c} \leq 7 \%$ or $>7 \%$ but can easily be stratified into any percentage based on clinical advise. Hypoglycemia was defined as a glucose value of $<70 \mathrm{mg} / \mathrm{dL}$. A severe hypoglycemic event was defined as an event requiring the assistance of another person to actively administer carbohydrate, glucagon, or other resuscitative actions in the presence of seizure or coma [20].

DySF is the daily weighted number of large monotonic glucose transitions that occur in less than one hour. DySF is derived from transition density profiles, described in [19], with the exception of employing equally-spaced bin thresholds for DySF analysis (Figure 1). First, raw CGM data were partitioned into $40 \mathrm{mg} / \mathrm{dL}$ bins and exact transition points were established (Figure 1a,b). Second, the magnitude of every continuous monotonic change in glucose bin levels was sorted into the number of thresholds crossed (e.g. 2, 4, -3, -5) (Figure 1c). Negative numbers indicate monotonic decreases and positive numbers indicate monotonic increases in glucose levels. Third, transitions were separated into the time interval necessary to complete each change (i.e. $<1 \mathrm{~h}$, between 1-2 h, 2-3 h, etc.). Finally, the frequency of each monotonic threshold crossing per day was plotted against the time interval needed to cross the indicated number of thresholds (Figure 1d).

DySF calculation has been added to the analytical software CGMGUIDE $^{\oplus}$ (patent-pending) as the weighted daily number of (> $|40| \mathrm{mg} /$
dL) monotonic transitions that occur within one hour [19] (Figure 1d). To calculate DySF, a patient's raw CGM data is first analyzed by the transition density profile method outlined above, using glucose bin threshold intervals of $40 \mathrm{mg} / \mathrm{dL}$. Monotonic transitions (i.e. periods of monotonic threshold crossings) that occur within one hour and that exceed a magnitude of 1 threshold $(40 \mathrm{mg} / \mathrm{dL})$ are identified. Each transition is assigned a magnitude equal to the number of thresholds crossed during that transition (For example, a monotonic decrease in glucose level across three thresholds would be given a magnitude of $-3)$. The sum of the absolute value of these scaled transition magnitudes is then divided by 24 to give the DySF units of weighted number of transitions per day (Figure 1d).

Robustness and sensitivity of DySF were evaluated for a range of choices in glucose threshold bin size $(5-100 \mathrm{mg} / \mathrm{dL})$ without observed improvement above the standard $40 \mathrm{mg} / \mathrm{dL}$ in correlation to $\mathrm{HE}$. Additionally, bin sizes of $40 \mathrm{mg} / \mathrm{dL}$ correspond to clinically important glycemic boundaries. The maximum glucose sampling interval at which DySF could be consistently measured was evaluated as $\leq 10$ minutes using pair-wise t-tests for $1 \mathrm{~min}, 5 \mathrm{~min}, 10 \mathrm{~min}, 15 \mathrm{~min}$, and 30 min intervals.

A logistic regression model was used to predict the likelihood of observing a severe hypoglycemic episode in the 6 months prior to CGM monitoring. Covariates considered were mean glucose, standard deviation of mean glucose, standard deviation of transition speeds, DySF, MAGE, MODD, and CONGA [1].

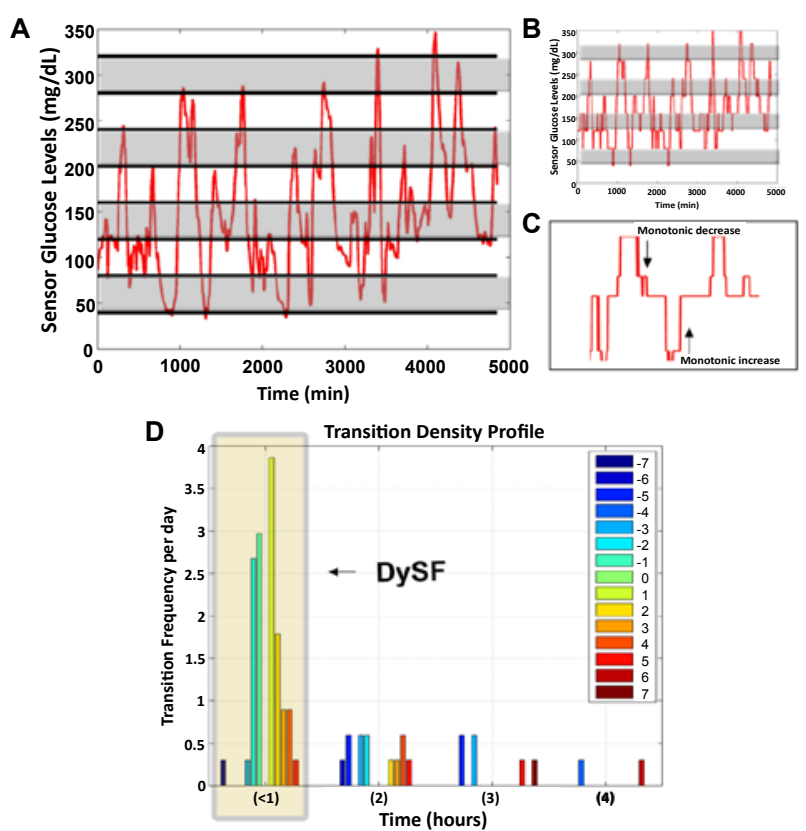

Figure 1: Calculation of DySF through Transition Density Profiles. A) Patient input glucose data, illustrating the $40 \mathrm{mg} / \mathrm{dL}$ increments in alternating grey and white. B) Smoothed input data in bins. C) Blow-up of data in (B) highlighting the monotonic changes to be recorded in the D) Transition Density Profile which compiles monotonic changes that occur in a specific time interval. The shaded region shows all the monotonic transitions that occurred in less than $1 \mathrm{~h}$. DySF is calculated as the sum of the number of transitions greater than one, weighted by the magnitude of the transition. (Example above: DySF $=0.3^{*}|-7|+0.3^{*}|-3|+$ $2.6^{*}|-2|+1.75^{\star}|2|+0.9^{*}|3|+0.9^{*}|4|+0.3^{*}|5|=19.5$. The weights listed as 0.3 and 2.6 for example are determined by the number of actual transitions occurred divided by the length of time the sample set is measured. Since there was one transition of -7 bins that occurred during the 5000 minutes or 3.4 days measured, we get $1 / 3.4=0.3$ to be our weighted factor for this transition. 
Citation: Rawlings RA, Yuan L, Shi H, Brehm W, Pop-Busui R et al. (2012) Dynamic Stress Factor (DySF): A Significant Predictor of Severe Hypoglycemic Events in Children with Type 1 Diabetes. J Diabetes Metab 3:177. doi:10.4172/2155-6156.1000177

Page 3 of 5

\section{Results}

\section{DySF as an indicator of Severe Hypoglycemia in children with type 1 diabetes}

The demographics and other clinical characteristics of this cohort were published in [20]. DySF was calculated at baseline for 441 T1DM patients where $53.02 \%$ were female, mean age was 24.6 years, and mean HbAlc was $7.4 \%$. These patients had an average DySF of $7.14 \pm 5.39$ with a cohort minimum at 0.33 and maximum at 54.89. Patients grouped by age and HbA1c had an average DySF of $7.00 \pm$ 3.90 (age 8-14), $8.78 \pm 7.50$ (age 15-24), $5.88 \pm 3.91$ (age $\geq 25$ ), $5.88 \pm$ $5.63(\mathrm{HbAlc} \leq 7)$, and $7.78 \pm 5.20(\mathrm{HbAlc}>7)$. In addition, to assess overall glycemic variability, CGM-GUIDE profiles were created for all patients to calculate the most widely used glycemic metrics and statistics discussed in Methods. A representative CGM-GUIDE profile that includes most widely used glycemic metrics and statistics is shown in Figure 2a.

To predict the occurrence of hypoglycemic episodes, simple and multiple logistic regression models were fitted to the data and all glycemic metrics were compared (Figure 2 a-c). Among the T1DM patients analyzed who had a mean glucose $\leq 140 \mathrm{mg} / \mathrm{dL}$ at baseline, DySF divided by the mean glucose (DySF/mean) was the best predictor of the frequency of hypoglycemic episodes ( $p$-value $=0.13$ ) (Figure $2 \mathrm{~b})$. The ratio DySF/mean was also the best predictor of frequency of hypoglycemic episodes in T1DM patients with a baseline HbA1c $\leq$ $6.5 \%(p$-value $=0.06)$ and in children aged $8-14$ years $(p$-value $=0.018)$ (Figure 2b).

Correlation between DySF and HbAlc, mean glucose, and other glycemic variability metrics showed low overlap of information between metrics (Figure 2c). Pearson correlation between DySF and each of the other measures of glycemic variability demonstrated that DySF provided additional information about glycemic variability with the exception of CONGA and standard deviation of slopes (Figure 2c).

We also found that in this cohort of T1DM patients, individuals within the same level of $\mathrm{HbAlc}$ values demonstrated widely different DySFs, or volatilities, indicating patient-specific HbAlc-independent variations in glucose transition times and/or dynamic ranges. In addition, individuals who exhibited similar HbA1c levels (including individuals with $\mathrm{HbAlc}$ below $7.0 \%$ ) had highly variable glucose volatility as measured by variable DySF. Individuals with the higher DySF values were those with poorer glycemic control as documented by HbA1c values larger than 7\% (Figure 3). When comparing patients of any age who had zero, one, or multiple severe hypoglycemic episodes, average DySF values decreased incrementally with increased incidence of severe HE, $7.27 \pm 5.6,6.63 \pm 4.23,5.61 \pm 3.47$, respectively.

Based on DySF values, a logistic model was used to predict the likelihood of children, 8-14, experiencing severe hypoglycemia. Children in the study who had the lowest DySF values (close to zero) had a $20 \%$ higher probability of having at least one severe hypoglycemic episode that required the assistance of another person for resuscitative actions (data not shown).

\section{Conclusion}

DySF is a new metric for the measurement of glycemic variability that measures the volatility of a patient's glucose dynamics by weighting the daily average of glucose transitions that occur in less than one hour. Usinglogistic regression models, DySF was found to be the most significant predictor of severe hypoglycemic episodes in children aged 8-14 years old, in patients with mean glucose less than or equal to $140 \mathrm{mg} / \mathrm{dL}$ (Figure $2 \mathrm{~b}$ ) and in patients with $\mathrm{HbAlc}<6.5 \%$. Lower
A

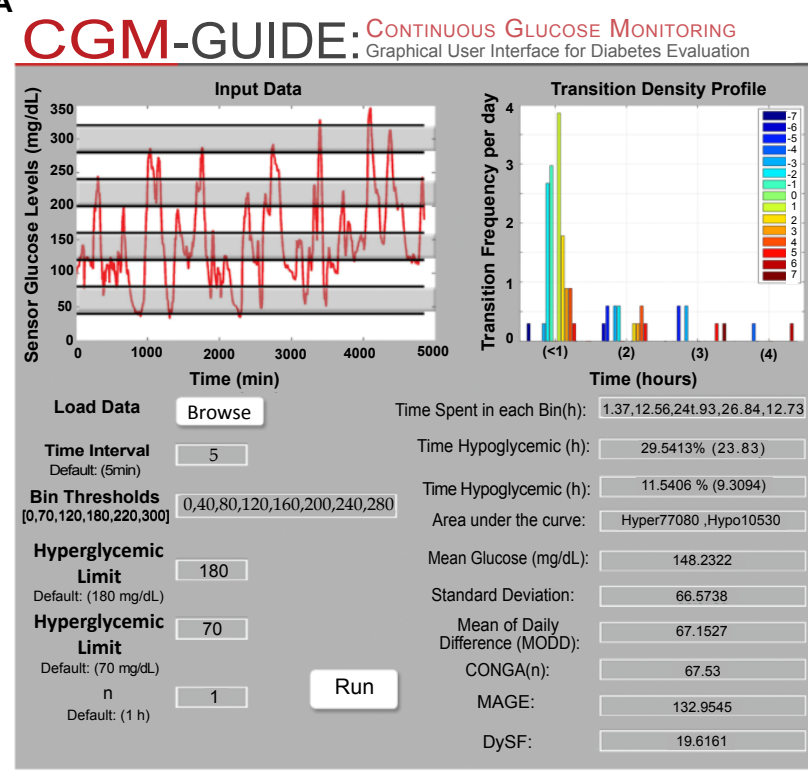

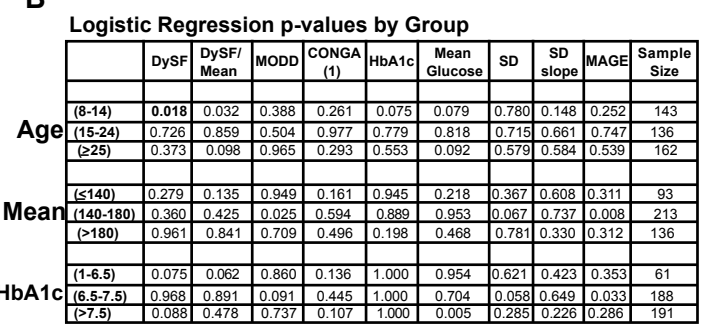

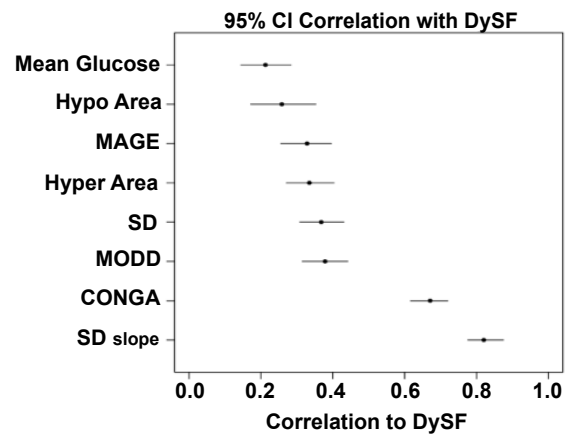

Figure 2: Dashboard of Glycemic Variability, Logistic Regression P-values, and Correlations of Previous Metrics to DySF. A) Glycemic Variability Profile for one patient with type 1 diabetes created by the CGM-GUIDE software [18]. Provides user adjustable bin thresholds, transition density profiles, statistics (mean, $\mathrm{SD}$, glycemic times/areas), and metric calculations including DySF, CONGA, MODD and MAGE. B) Table of p-values from individual logistic regressions to predict the number of hypoglycemic episodes (HE) based on the described metrics. DySF shows the most predictive power among metrics in children $8-14$ yr ( $p$-value $=$ 0.018). C) Correlation of previous statistics and metrics to DySF. Confidence intervals were obtained as the central $95 \%$ of correlation coefficients observed on the basis of 10,000 bootstrap samples (of size equal to the original sample). Dots represent the means of the resulting empirical distributions and are essentially equivalent to the one sample point estimates from the original data. 

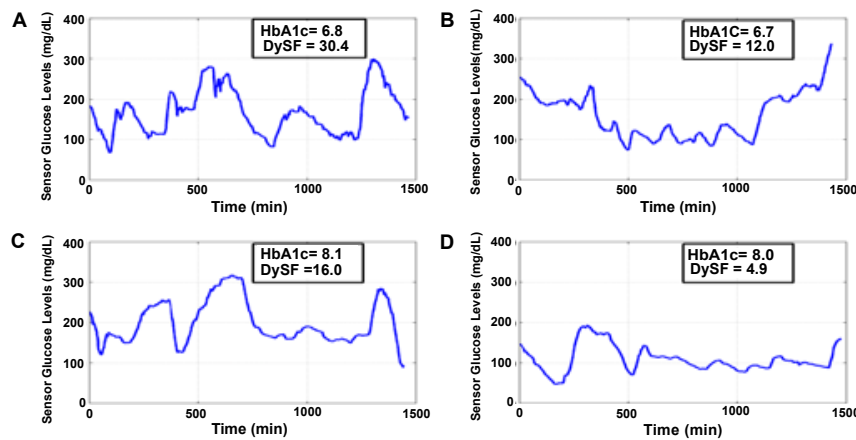

Figure 3: DySF Distinguishes Patients into Visually Distinct Classes Independent of HbA1c Level. A-B) Two individuals within the same HbA1c level $(<7)$ whose volatility is separated by DySF. C-D) Two individuals within the same $\mathrm{HbA} 1 \mathrm{c}$ level $(>7)$ whose volatility is separated by DySF

DySF values corresponded to higher risk of hypoglycemia and were indicative of smaller and/or slower glucose transitions over time.

Several insights can be drawn from these results. First, we found that DySF/mean is a sensitive tool that can more favorably assess and predict patients' risk for experiencing severe hypoglycemic episodes compared to $\mathrm{HbAlc}$ alone. We also confirm in a large sample of T1DM patients that low HbAlc levels can be misleading as an indicator of glycemic control. Patients with HbAlc below $6.5 \%$ are traditionally considered to have "well-controlled" diabetes [1]. We demonstrate in this cohort, that a lower $\mathrm{HbAlclevel}(\leq 6.5 \%)$ may be the result of a high incidence of hypoglycemia as opposed to tighter glycemic control. We also show that by using DySF/mean, we are able to significantly enhance our ability to predict severe hypoglycemic events in patients with either low $\mathrm{HbA1c}$, low mean BG and in children. This has very important clinical significance as it helps create a patient specific phenotype that can be used by clinicians to preventsevere hypoglycemic events.

Second, the association between DySF and the occurrence of severe hypoglycemic episodes was found to be most significant in children (aged 8-14 years) across all levels of HbAlc (Figure 2b). Subjects were observed to vary widely in DySF values within the same class of $\mathrm{HbAlc}$, suggesting the degree of glucose fluctuations to be independent of HbA1c level. This allows DySF to provide a different perspective on glycemic variability from what $\mathrm{HbA} 1 \mathrm{c}$ measures-namely, volatility.

Some studies have evaluated how children with T1DM identify severe hypoglycemic episodes [20], which is critical for their prevention. Gonder-Fredrick et al. demonstrated that children with type 1 diabetes failed to recognize greater than $40 \%$ of hypoglycemic occurrences, and Meltzer et al. observed that the average adolescent patient made irrelevant or inaccurate glucose estimations greater than $61 \%$ of the time [21,22]. Because HE often occur during times when patients fail to recognize symptoms associated with hypoglycemia, DySF can be used to assess the severity and speed of glycemic excursions and therefore can be an effective clinical tool to prevent $\mathrm{HE}$ and its serious consequences.

Lastly, the correlation between DySF and other glycemic variability metrics is relatively low, with often less than $50 \%$ of the variation in DySF being accounted for by other metrics (Figure 2c). This suggests that a combination of existing glycemic variability metrics and DySF will be best suited to assess different populations and/or varying disease complications. A recent study by Guerra et al. explored rates of change of glucose, using a deconvolution algorithm that introduces uncertainty in the model parameters and considers less than 30 minutes of glucose history to predict future risk [23]. Previous glucose variability metrics, such as MODD, are considered to be a measure of daily glucose consistency [16], MAGE a measure of fluctuation severity, and CONGA a measure of glucose lability [16], or the likelihood of undergoing any change in glucose level over a defined length of time. By introducing DySF, a measure of glucose volatility, we can now explore over much longer historiesof BG data, the long-term effects of changes in glucose speed and magnitude on patient outcomes. DySF therefore increases significantly the predictive power for hypoglycemia and other complications. In addition, we demonstrate that DySF offers tailored information about specific populations, such as children (8-14 years), or patients with various $\mathrm{HbA} 1 \mathrm{c}$ levels.

Despite clear differences in their defining properties, to date existing glucose variability metrics are used either interchangeably or individually with statistics such as SD to assess overall glycemic variability. Cameron et al. demonstrated that glucose variability metrics, though correlated with each other in non-diabetic patients, are not correlated in diabetic populations [9]. Clarke and Kovatchev [24,25] have applied metrics for studying hypoglycemic events in patients using single monitor blood glucose (SMBG) measurements. Their studies show some predictive measures of future hypoglycemic events but the metrics were strongly correlated to the past history of time spent in lower glycemic ranges and did not consider the entire course of patient data which includes hypo and hyper regions as well as normal ranges. Thus, studies in diabetic populations that look at only one or two measures, or less sensitive measures of glucose variability, cannot comprehensively assess glycemic variability because these do not take into account the full range of glycemic states a patient may encounter over shorter or longer periods of time, nor the spectrum of transition profiles from these states [26-28], whereas glucose variability profiles such as those generated by CGM-GUIDE overcome this challenge.

DySF and CGM-GUIDE profiles may also prove to be superior to current individual metrics in evaluating the role of glucose variability in the development of chronic diabetes complications [19]. At present, low DySF is important in assessing trends in hypoglycemia; however, high volatility may become important when assessing chronic diabetes complications and disease progression.

Rapid glucose fluctuations have been hypothesized to incorporate "stress" into a patient's system by increasing oxidative stress and contributing to the development of microvascular complicationsand cardiovascular disease $[3-5,8,29,30]$. However, others have questioned this concept [31]. The long-term effect of sustained volatility is the next pressing question in developing an improved picture of diabetes progression toward chronic conditions, especially in what are currently considered well-controlled populations. Towards this effort, researchers and clinicians are now able to apply DySF, in conjunction with $\mathrm{HbAlc}$, as a tool to enhance their ability to understand type 1diabetes and to procure treatment options.

\section{Acknowledgments}

We thank James Henderson for his helpful discussion, and editing.These studies were supported by the NSF-DMS 0634590 to Dr. Patrick Nelson, 1R01HL102334-01 to Dr. Pop-Busui, NSF-AGEP to Dr. Renata Rawlings, and the UM-SUBMERGE undergraduate research scholarships to Lo-Hua Yuan and Hang Shi. No potential conflicts of interest relevant to this article were reported.

R.A.R. participated in research design, performance of research, data analysis, and writing the manuscript. L.Y. participated in performance of research, data analysis and editing the manuscript. H.S. participated in data analysis and writing and editing the manuscript. W.B. participated in research design. R.P. participated in research design, and editing the manuscript. P.W.N. participated in research design, performance of research and writing the manuscript. 
Citation: Rawlings RA, Yuan L, Shi H, Brehm W, Pop-Busui R et al. (2012) Dynamic Stress Factor (DySF): A Significant Predictor of Severe Hypoglycemic Events in Children with Type 1 Diabetes. J Diabetes Metab 3:177. doi:10.4172/2155-6156.1000177

\section{References}

1. (1995) The relationship of glycemic exposure ( $\mathrm{HbA} 1 \mathrm{c})$ to the risk of development and progression of retinopathy in the diabetes control and complications trial. Diabetes 44: 968-983.

2. (1985) U.K. prospective diabetes study. II. Reduction in HbA1c with basa insulin supplement, sulphonylurea, or biguanide therapy in maturity-onset diabetes. A multicenter study. Diabetes 34: 793-798.

3. Hirsch IB, Brownlee M (2010) Beyond hemoglobin A1c--need for additional markers of risk for diabetic microvascular complications. JAMA 303: 2291 2292.

4. Su G, Mi S, Tao H, Li Z, Yang H, et al. (2011) Association of glycemic variability and the presence and severity of coronary artery disease in patients with type 2 diabetes. Cardiovasc Diabetol 10: 19.

5. Dungan KM, Binkley P, Nagaraja HN, Schuster D, Osei K (2011) The effect of glycaemic control and glycaemic variability on mortality in patients hospitalized with congestive heart failure. Diabetes Metab Res Review 27: 85-93.

6. Brownlee M, Hirsch IB (2006) Glycemic variability: a hemoglobin A1cindependent risk factor for diabetic complications. JAMA 295: 1707-1708.

7. Ceriello A, Ihnat MA (2010) 'Glycaemic variability': a new therapeutic challenge in diabetes and the critical care setting. Diabet Med 27: 862-867.

8. Zaccardi F, Pitocco D, Ghirlanda G (2009) Glycemic risk factors of diabetic vascular complications: the role of glycemic variability. Diabetes Metab Res Rev 25: 199-207.

9. Cameron FJ, Donath SM, Baghurst PA (2010) Measuring glycaemic variation Curr Diabetes Rev 6: 17-26.

10. Weber C, Schnell O (2009) The assessment of glycemic variability and its impact on diabetes-related complications: an overview. Diabetes Technol Ther 11: 623-633.

11. Hirsch IB, Brownlee M (2005) Should minimal blood glucose variability become the gold standard of glycemic control? J Diabetes Complications 19: 178-181.

12. Clarke W, Kovatchev B (2009) Statistical tools to analyze continuous glucose monitor data. Diabet Technol Ther 11: S45-54.

13. Nalysnyk L, Hernandez-Medina M, Krishnarajah G (2010) Glycaemic variability and complications in patients with diabetes mellitus: evidence from a systematic review of the literature. Diabetes Obes Metab 12: 288-298.

14. Service FJ, Molnar GD, Rosevear JW, Ackerman E, Gatewood LC, et al. (1970) Mean amplitude of glycemic excursions, a measure of diabetic instability. Diabetes 19: 644-655.

15. Molnar GD, Taylor WF, Ho MM (1972) Day-to-day variation of continuously monitored glycaemia: a further measure of diabetic instability. Diabetologia 8 : 342-348.

16. McDonnell CM, Donath SM, Vidmar SI, Werther GA, Cameron FJ (2005) A novel approach to continuous glucose analysis utilizing glycemic variation. Diabetes Technol Ther 7: 253-263.
17. Whitelaw BC, Choudhary P, Hopkins D (2011) Evaluating rate of change as an index of glycemic variability, using continuous glucose monitoring data. Diabetes Technol Ther 13: 631-636.

18. Sivananthan S, Naumova V, Man CD, Facchinetti A, Renard E, et al. (2011) Assessment of blood glucose predictors: the prediction-error grid analysis. Diabetes Technol Ther 13: 787-796.

19. Rawlings RA, Shi H, Yuan LH, Brehm W, Pop-Busui R, et al. (2011) Translating Glucose Variability Metrics into the Clinic via Continuous Glucose Monitoring: a Graphical User Interface for Diabetes Evaluation (CGM-GUIDE ${ }^{\odot}$ ). Diabetes Technol Ther 13: 1241-1248.

20. JDRF CGM Study Group (2008) JDRF randomized clinical trial to assess the efficacy of real-time continuous glucose monitoring in the management of type 1 diabetes: research design and methods. Diabetes Technol Ther 10: 310-321.

21. Gonder-Frederick L, Zrebiec J, Bauchowitz A, Lee J, Cox D, et al. (2008) Detection of hypoglycemia by children with type 1 diabetes 6 to 11 years of age and their parents: a field study. Pediatrics 121: e489-495.

22. Meltzer LJ, Johnson SB, Pappachan S, Silverstein J (2003) Blood glucose estimations in adolescents with type 1 diabetes: predictors of accuracy and error. J Pediatr Psychol 28: 203-11

23. Guerra S, Sparacino G, Facchinetti A, Schiavon M, Man CD, et al. (2011) A dynamic risk measure from continuous glucose monitoring data. Diabetes Technolo Ther 13: 843-852.

24. Cos DJ, Gonder-Frederick L, Ritterband L, Clarke W, Kovatchev B (2007) Prediction of severe hypoglycemia. Diabetes Care 30: 1370-1373.

25. Kovatchv B, Cox DJ, Gonder-Frederick LA, Young-Hyman D, Schlundt D, et al. (1998) Assessment of risk for severe hypoglycemia among adults with IDDM Diabetes Care 21: 1870-1875.

26. Bruttomesso D, Crazzolara D, Maran A, Costa S, Dal Pos M, et al. (2008) In Type 1 diabetic patients with good glycaemic control, blood glucose variability is lower during continuous subcutaneous insulin infusion than during multiple daily injections with insulin glargine. Diabet Med 25: 326-332.

27. Kilpatrick ES, Rigby AS, Goode K, Atkin SL (2007) Relating mean blood glucose and glucose variability to the risk of multiple episodes of hypoglycaemia in type 1 diabetes. Diabetologia 50: 2553-2561.

28. White NH, Chase HP, Arslanian S, Tamborlane WV (2009) Comparison of glycemic variability associated with insulin glargine and intermediate-acting insulin when used as the basal component of multiple daily injections for adolescents with type 1 diabetes. Diabetes Care 32: 387-393.

29. Monnier L, Mas E, Ginet C, Michel F, Villon L, et al. (2006) Activation of oxidative stress by acute glucose fluctuations compared with sustained chronic hyperglycemia in patients with type 2 diabetes. JAMA 295: 1681-1687.

30. Kilpatrick ES, Rigby AS, Atkin SL (2009) Effect of glucose variability on the long-term risk of microvascular complications in type 1 diabetes. Diabetes Care 32: 1901-1903

31. Ramakrishna V, Jailkhani R (2007) Evaluation of oxidative stress in insulin dependent diabetes mellitus (IDDM) patients. Diagn Pathol 2: 22. 